\title{
Efectos de la extracción forestal sobre la estructura y composición de un bosque pluvial del Pacífico colombiano
}

\author{
Effects of logging on structure and composition of a rain forest \\ in the Colombian Pacific
}

\author{
Nadia Bonilla-Luna*, Harvency Cuesta-Córdoba*, Hamleth Valois-Cuesta*
}

\begin{abstract}
Resumen
Se describen los impactos más evidentes de la tala selectiva de árboles sobre la estructura y composición de un bosque pluvial tropical de tierras bajas en el corregimiento de San Isidro (Chocó, Colombia). Se realizaron inventarios de vegetación en 10 parcelas de $2 \times 50$ metros ( 0.1 ha) ubicadas dentro de un bosques no intervenido y en otras 10 parcelas de similares dimensiones pero ubicadas en un bosque usado para extraer madera selectivamente. Se registró un mayor número de especies, géneros y familias de plantas leñosas en el bosque natural que en el bosque afectado por la extracción selectiva de maderas; también los elementos arbóreos que dominan el dosel, como también el número de especies y géneros de las 10 familias más representativas encontradas en el bosque natural, se vieron reducidos en el bosque afectado por la tala selectiva. Este proceso de reducción de la complejidad estructural del bosque en el área de estudio, seguro redunda negativamente en la dinámica de las poblaciones de fauna que en ellos habita, sobre todo aquellos animales dependientes de los estratos superiores del bosque.
\end{abstract}

Palabras clave: Bosque pluvial tropical de tierras bajas; Chocó; Colombia; San Isidro;

Tala selectiva.

\begin{abstract}
Herein, we described the most evident effects of selective logging on the structure and composition of a lowland tropical rain forest in San Isidro, Chocó, Colombia. Structure and composition of woody elements from 10 plots $(2 \times 50$ meters $=0.1$ ha) located in a natural forest and 10 plots $(2 \times 50$ meters $=0.1 \mathrm{ha}$ ) located in a forest used for selective logging was analyzed. We report a greater number of species, genera and families of woody plants in the natural forest than in forest with selective logging. In addition, forest where selective logging takes place experienced a significant reduction in number of canopy-dominant trees, as well as in number of species and genera of the 10 more representative families found in the analyzed natural forest. We predict that this documented reduction in the natural forest structural complexity will have a deleterious impact on the population dynamics of fauna in the study area, particularly on canopy-dependent fauna.
\end{abstract}

Keywords: Chocó; Colombia; Lowland tropical rainforests; San Isidro; Selective logging.

\section{Introducción}

Los bosques pluviales tropicales de tierras bajas poseen alta biodiversidad y son vitales en los ciclos globales de agua y carbono; además, poseen una de las más altas biomasas dentro de los ecosistemas continentales (Sarmiento et al. 2005). Este panorama se está modificando entre otras razones, por la explotación forestal bajo esquemas de manejo no acordes con la realidad ecológica de cada región (Johnson y Cabarle, 1993). Al respecto, Bryant et al. (1997) comentan que la explotación forestal de bos-

* Programa de Biología con Énfasis en Recursos Naturales, Facultad de Ciencias Básicas, Universidad Tecnológica del Chocó, Quibdó,Colombia.e-mail: havalois@yahoo.com nabolu35@hotmail.com

Fecha recepción: Octubre 1, $2010 \quad$ Fecha aprobación: Marzo 4, 2011 
ques naturales constituye una de las principales amenazas para la conservación de la diversidad biológica en la región tropical. Algunos autores argumentan que la extracción de maderas de bosques naturales reduce la complejidad estructural de la vegetación (Cannon et al. 1994; Ochoa, 1998; Padmawathe et al. 2004), induce cambios en la adecuación de las comunidades de animales silvestres (Johns, 1988; Aleixo, 1999; Ochoa, 2000; Bin Hussin y Francis, 2001; Ghazoul y Hill, 2001; Ochoa y Soriano, 2001; Plumptre y Grieser, 2001; Soriano y Ochoa, 2001; Vitt y Caldwell, 2001) y genera reducción de las fuentes de agua, fragmentación del hábitat y erosión de los suelos (Laurance et al. 2001).

Dentro de los bosques neotropicales, los bosques pluviales de tierras bajas en el departamento del Chocó (Pacífico colombiano) poseen alta riqueza de especies y endemismos; se estima que los mismos podrían albergar entre 7000 y 8000 especies de plantas superiores (Forero y Gentry, 1989), muchas de ellas aún desconocidas y posiblemente promisorias. En el Chocó, la explotación de maderas en bosques naturales (una de las principales actividades económicas) constituye una amenaza para la permanencia de la biodiversidad y equilibrio dinámico de los ecosistemas forestales regionales a largo plazo.

En este trabajo, se describen algunos de los efectos más evidentes de la extracción selectiva de maderas sobre la estructura y composición de una selva pluvial de tierras bajas en el corregimiento de San Isidro (Río Quito, Chocó), con el propósito de aportar información que redunde en la implementación de planes de manejo y conservación de bosques naturales en el Pacífico colombiano.

\section{Materiales y métodos}

Área de estudio. El trabajo se desarrolló en el corregimiento de San Isidro, municipio de Río Quito (Chocó, Colombia). San Isidro (5 $\left.5^{\circ} 25^{\prime} \mathrm{Ny} 76^{\circ} 40^{\prime} \mathrm{W}\right)$ se encuentra a $45 \mathrm{msnm}$ y según el sistema de Holdrige (1982) corresponde a la zona de vida bosque pluvial tropical (bp-T), que se caracteriza por presentar una temperatura mayor a $24^{\circ} \mathrm{C}$ y precipitación entre 8,000 $10,000 \mathrm{~mm}$ anual. Una de las principales actividades económicas en la zona es la explotación forestal de bosques naturales. Sin embargo, se realiza minería, pesca y agricultura a baja escala.
Métodos. En el año 2006 se realizaron censos de vegetación en dos áreas boscosas:

a. Quebrada «Madre Vieja»: no presentaba ningún grado aparente de intervención antrópica y

b. Quebrada «El Chontaduro»: era una zona que había sido usada para extracción selectiva de maderas hacia tres años (com. pers. aserradores de la zona).

Las dos áreas distaban $17 \mathrm{~km}$ aproximadamente una de otra y ambas se ubicaban dentro de la unidad de paisaje de zona aluvial. En las dos áreas objeto de estudio, se establecieron al azar diez parcelas de $2 \times 50$ $\mathrm{m}(0,1 \mathrm{ha})$ siguiendo los lineamientos de Villareal et al. (2004). En cada parcela, se censaron los individuos leñosos (excluyendo epífitos) con diámetro a la altura del pecho (DAP) $>1 \mathrm{~cm}$, registrando su DAP, altura total, nombre científico y familia. El material colectado se llevó al herbario «CHOCO» de la Universidad Tecnológica del Chocó, donde se identificó por medio de confrontación con otras muestras y la ayuda de literatura especializada y guías ilustradas (Gentry, 1993; Mahecha, 1997).

\section{Resultados y discusión}

Características del aprovechamiento maderero en San Isidro (Chocó). Las especies con madera de valor comercial que frecuentemente se extraen del bosque son Sacoglottis procera, Guarea sp., Brosimum utile, Virola sebifera, Himatanthus sp., Manilkara sp., Eschweilera pittieri, Hymenaea palustris, Aniba sp., Aniba perutilis, Lacmellea sp. y Licania durifolia. Prácticamente, se talan árboles de éstas y otras especies menos comerciales durante todo el año, siendo la motosierra $(70 \%)$ y el hacha $(30 \%)$ las principales herramientas empleadas para tal fin. Los árboles talados son preferiblemente los de mayor porte (DAP, altura y rectitud del fuste) (Figura 3). Una vez talado el árbol, la madera se acumula en zonas de acopio al interior del bosque y luego se transporta en mulas por una serie de caminos que conectan con quebradas o carreteras.

Cambios en la estructura florística. La matriz fisionómica predominante del bosque natural, donde la vegetación es interrumpida solamente por la formación de claros naturales o pequeños lechos de quebradas, evidentemente se transforma en un paisaje con poca dominancia de elementos que confor- 


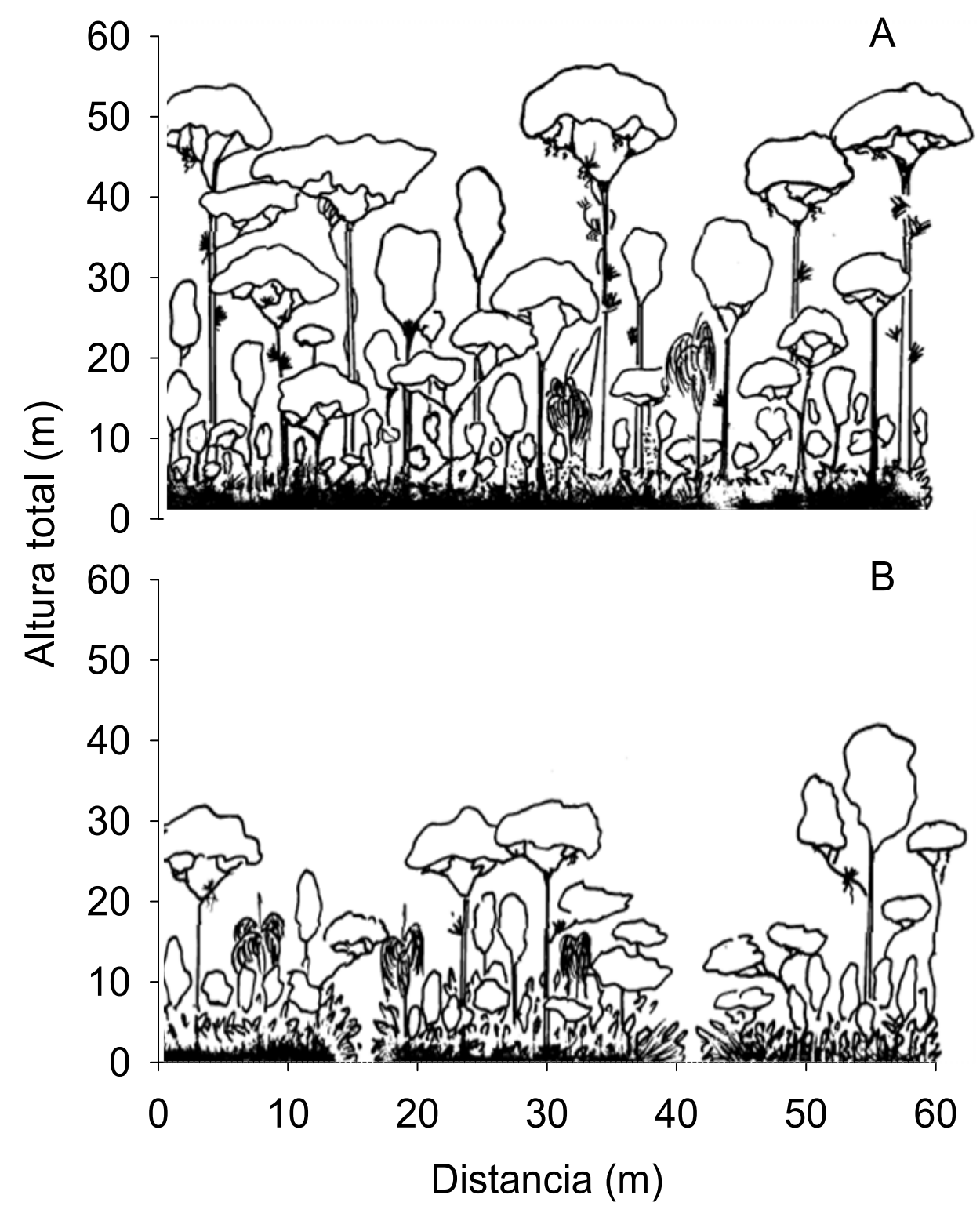

Figura 1. Perfiles esquemáticos de la vegetación en San Isidro (Chocó). A. Bosque natural sin registro de intervención antrópica. B. Bosque con extracción selectiva de maderas.

man el dosel y alta abundancia de plantas de estratos inferiores (Figura 1, A vs. B; Figura 3). Esta transformación, se debe sobre todo a la tala selectiva de árboles de valor comercial de gran porte (Figura 2A), y a la consiguiente apertura de caminos y zonas de acopio para las trozas de madera (Figura 2B).

La apertura de caminos, vías de arrastre y patios de acopio promueven la creación de diferentes microhábitats asociados con altos niveles de luz, suelos compactados y altas temperaturas superficiales que ofrecen oportunidades limitadas para el estable- cimiento de especies arbóreas (Uhl y Vieira, 1989; Pinard et al. 1996). Carrera y Pinelo (1995), Guzmán y Cordero (1996) y Jackson et al. (2000) consideran que en bosques usados para la extracción de maderas, después de la construcción de caminos, el arrastre de maderos es la operación que más impacto genera sobre el suelo. Así, los disturbios en el suelo constituyen uno de los principales factores que limitan la regeneración de especies arbóreas en bosques aprovechados forestalmente (Van Gardingen et al. 1998).

En las parcelas ubicadas en el área donde el bos- 

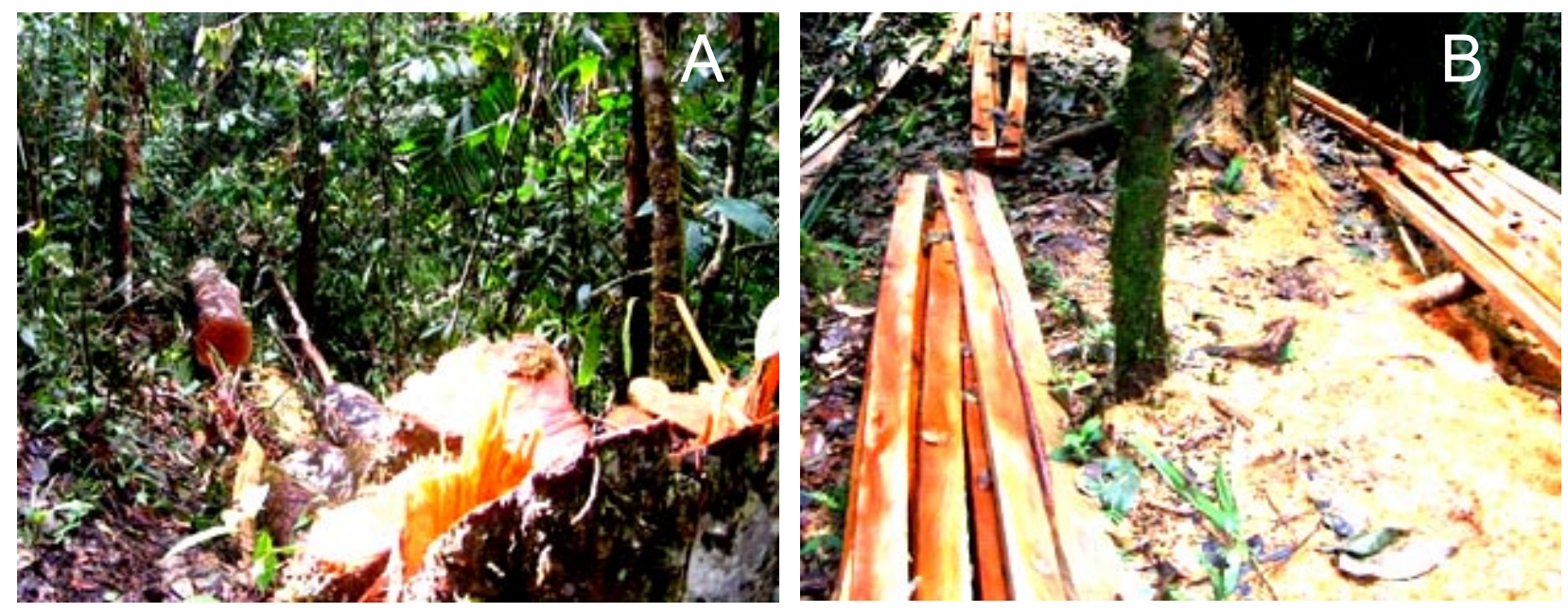

Figura 2. Panorámica de las áreas aprovechadas forestalmente en San Isidro:

A. Árbol de gran porte derribado. B. Zona para el acopio y distribución de madera.

que ha sido afectado por tala selectiva de árboles, con frecuencia se observaron árboles rotos en pie y una baja frecuencia de epífitas vasculares, caso contrario a lo apreciado en las parcelas censadas en zonas no afectadas por tala selectiva. Padmawathe et al. (2004) registraron una reducción significativa en la riqueza y abundancia de epífitas vasculares (excepto orquídeas) en un bosque húmedo de tierras bajas sometido a explotación en la India; ellos atribuyen esta reducción a alteraciones en los microclimas, características de los sustratos y cobertura del dosel. Según Ochoa (1998), los árboles de gran porte al caer generan daños mecánicos a la vegetación arbórea circundante, promoviendo además, el arrastre de elementos trepadores que interconectan la masa boscosa.

Cambios en la composición florística. En las parcelas ubicadas en bosque natural se registraron 33 familias, 59 géneros y 87 especies; mientras que en aquellas afectadas por extracción de maderas, se enconraron 29 familias, 55 géneros y 67 especies. Lauraceae, Rubiaceae, Annonaceae, Sapotaceae y Euforbiaceae fueron las familias más representativas (riqueza de géneros y especies) en zonas naturales, mientras que Melastomataceae, Clusiaceae, Apocynaceae, Myristicaceae y Lecitydaceae dominaron en zonas que habían sido sometidas a extracción de maderas (Figura 4). Los géneros Licania (tres especies), Ocotea (dos), Guatteria (dos), Protium (dos) y Compsoneura (dos) fueron más ricos en el bosque natural, mientras que Miconia (cinco especies), Ossaea (dos), Carapa (dos) y Tovomitopsis (dos) parecen ser propios de zonas intervenidas. Especies pertenecientes a las familias Melastomataceae y Myristicaceae como también a los géneros Ossaea y Miconia se han registrado con frecuencia en otros bosques del Chocó sometidos a algún tipo de intervención (Mosquera y Mosquera, 2002; Córdoba y González, 2005). En el área de estudio, las especies de la familia Lauraceae en especial de los géneros Aniba y Ocotea, son frecuentemente taladas debido a la calidad atribuida a su madera. Arrollo et al. (1994) apreciaron en esta misma zona de estudio, una reducción notable de especies maderables de importancia económica y la dominancia relativa de especies arbustivas cuya madera posee poco valor en el mercado.

Un breve análisis sobre los efectos de la tala selectiva de árboles en San Isidro (Chocó). Las actividades relacionadas con la extracción de maderas en el área de estudio (tala de árboles de gran porte, apertura de grandes claros para acopio de maderos y construcción de caminos para el transporte de los mismos), aunque aparentemente están vinculadas a pocas especies de valor comercial, al parecer generan una serie de efectos importantes sobre la vegetación natural, tales como: reducción de los elementos arbóreos característicos de los estratos superiores y cambios en la riqueza taxonómica de algunos grupos de plantas dominantes. Este proceso de transformación de la vegetación, podría estar determinando variaciones importantes en la diná- 


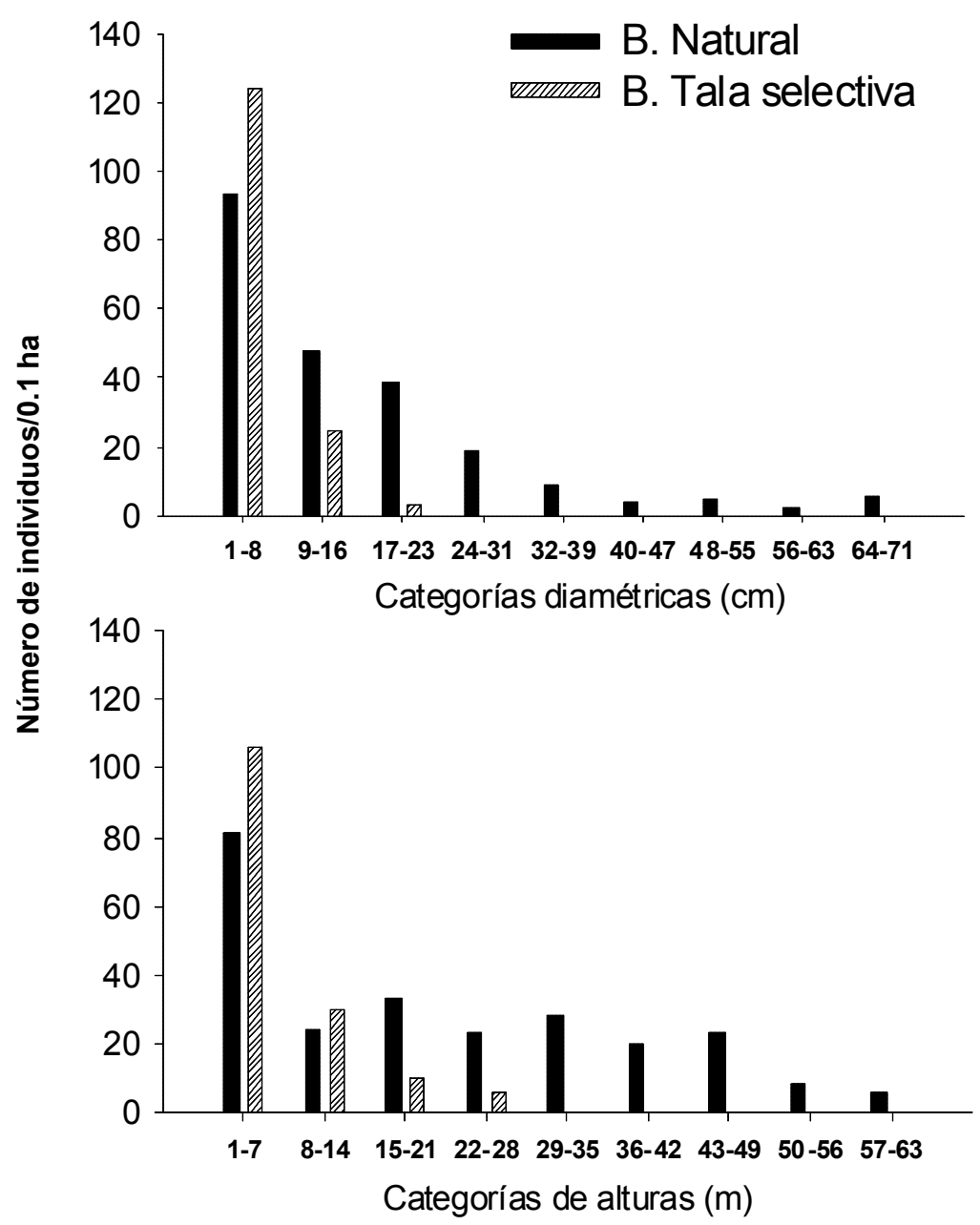

Figura 3. Distribución de individuos en categorías diamétricas y alturas totales en bosques no intervenidos (B. natural) y en bosques afectados por extracción selectiva de maderas (B. tala selectiva) en San Isidro, río Quito, Chocó, Colombia.

mica de estos ecosistemas y en la adecuación de las poblaciones de fauna silvestre que allí conviven. Según algunos autores, los cambios en la estructura de la vegetación inducidos por la explotación forestal, podrían redundar en un menor aporte de recursos fundamentales para animales silvestres, sobre todo aquellas especies dependientes de los estratos superiores del bosque (Johns, 1988, 1992; Thiollay, 1992; Frumhoff 1995; Mason, 1996; Ochoa, 1997; Bin Hussin y Francis, 2001; Ghazoul y Hill, 2001; Ochoa y Soriano, 2001; Plumptre y Grieser, 2001; Soriano y Ochoa, 2001). Los resultados de este trabajo sopor$\tan$ los reportes de otros autores, en los que se plantea que la extracción de maderas desde bosques naturales genera cambios sustanciales sobre su estructura y composición, y la consecuente pérdida de hábitats y recursos indispensables para la supervivencia de la fauna que en ellos habita, especialmente aquella que depende del dosel (Uhl y Vieira, 1989; Cannon et al. 1994, Killeen et al. 1998; Ochoa 1998; Fredericksen 2000; Padmawathe et al. 2004). Serán necesarias futuras investigaciones para comprender mejor los efectos del uso forestal de los bosques naturales del Chocó, en función de buscar las mejores herramientas y técnicas que permitan su aprovechamiento y conservación. En este sentido, resultará de especial interés evaluar cómo diferentes tratamientos selvícolas pueden afectar la regeneración natural de especies clave y/o grupos funcionales, y si ello puede generar diversas rutas sucesionales hacia la etapa clímax de la vegetación. 


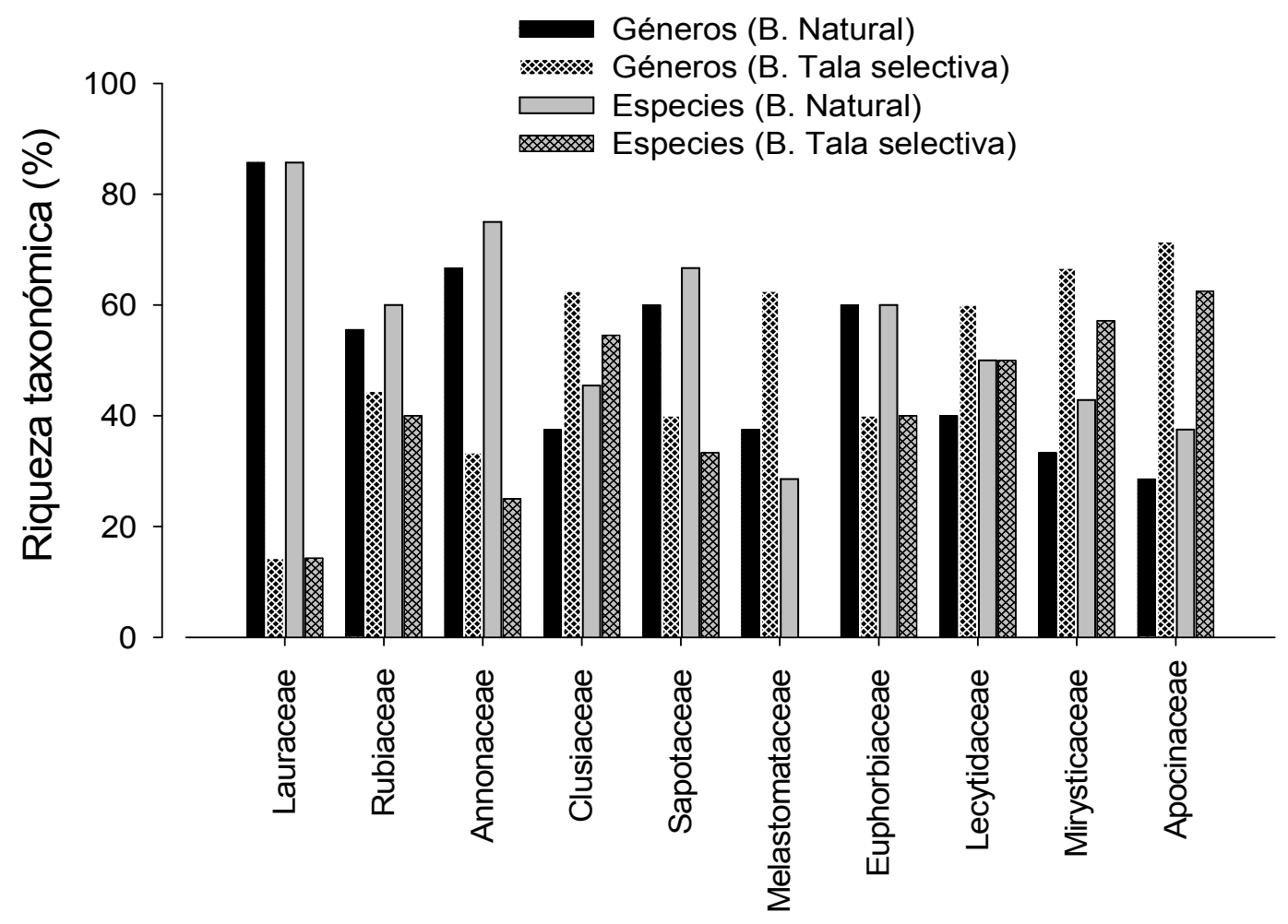

Figura 4. Patrones de riqueza de las diez familias más representativas en bosques no intervenidos (B. Natural) y en bosques afectados por extracción selectiva de maderas (B. Tala selectiva) en San Isidro, río Quito, Chocó, Colombia.

\section{Agradecimientos}

A la comunidad de San Isidro su colaboración en campo, a Leonardo Palacios su ayuda en la identificación de material vegetal, a Yan A. Ramos, Franklin Barrios, Yuber Palacios y dos revisores anónimos sus comentarios. Esta investigación fue acreditada por el Programa de Biología con Énfasis en Recursos Naturales de la Universidad Tecnológica del Chocó.

\section{Literatura citada}

Alexandre A. 1999. Effects of selective logging on a bird community in the Brazilian Atlantic forest. Condor. 101 (3): 537-48.

Arroyo JE, Palacios J, Mena A, Mosquera M, Ramos Y, Mosquera A, et al. 1994. Estructura de un bosque pluvial tropical (bp-T) intervenido (explotación selectiva de maderas). En: Memorias del Primer Congreso Nacional sobre Biodiversidad. Biopacífico. Cali: Universidad del Valle, Instituto de Estudios del Pacífico. p. 15-119.

Bin Hussin MZ, Francis CM. 2001. The effects of logging on birds in tropical forests of indo-Australia. En: Fimbel
RA, Grajal A, Robinson JG. (Eds.). The cutting edge: conserving wildlife in logged tropical forest. New York: Columbia University Press. p. 193-238.

Bryant D, Nielsen D, Tangley L. 1997. The last frontier forests: ecosystems \& economies on the edge: what is the status of the world's remaining large, natural forest ecosystems? Washington, DC: World Resources Institute. 42 p.

Cannon CH, Peart DR, Leighton M, Kartawinata K. 1994. The structure of lowland rainforest after selective logging in West Kalimantan, Indonesia. Forest Ecol Manag. 67(13): 49-68.

Carrera F, Pinelo G. 1995. Prácticas mejoradas para aprovechamientos forestales de bajo impacto. Informe Técnico $\mathrm{N}^{\mathrm{o}}$ 262. Turrialba: CATIE/CONAP. $61 \mathrm{p}$.

Córdoba AJ, González D. 2005. Regeneración natural en claros de un bosque pluvial tropical en Pacurita, Chocó, Colombia (Trabajo de Grado). Quibdó: Programa de Biología con Énfasis en Recursos Naturales, Universidad Tecnológica del Chocó. 111 p.

Fimbel RA, Grajal A, Robinson JG. 2001. The cutting edge: conserving wildlife in logged tropical forest. New York: Columbia University Press. 808 p.

Forero E, Gentry A. 1989. Lista anotadas de plantas del departamento del Chocó. Bogotá: Instituto de Ciencias Naturales, Museo de Historia Natural. 143 p.

Fredericksen TS. 2000. Logging and conservation of tropical 
forests in Bolivia. Int For Rev. 2 (4): 271-8.

Frumhoff PC. 1995. Conserving wildlife in tropical forests managed for timber. BioScience. 45 (7): 456-64.

Gentry A. 1993. A field guide to the families and genera of woody plants of northwest South America (Colombia, Ecuador, Perú). Washington DC: Conservation International. $895 \mathrm{p}$.

Ghazoul J, Hill J. 2001. The impacts of selective logging on tropical forest invertebrates. En: Fimbel RA, Grajal A, Robinson JG. (eds.). The cutting edge: conserving wildlife in logged tropical forest. New York: Columbia University Press. p. 260-303.

Guzmán R, Cordero W. 1996. Manejo sostenible de bosques: una alternativa a los problemas actuales. En: BOLFOR. Hacia el manejo forestal sostenible. Santa Cruz: Ministerio de Desarrollo Sostenible y Medio Ambiente de Bolivia. p. 179-205.

Holdridge LR. 1996. Ecología basada en zonas de vida. Colección Libros y Materiales Educativos $N^{\circ} 83$. San José: IICA. 216 p.

Jackson SM, Fredericksen TS, Malcolm JR. 2000. Evaluación de los disturbios y daños causados al bosque residual durante el aprovechamiento por selección en un bosque tropical de Bolivia. Documento Técnico 91. Proyecto BOLFOR. Santa Cruz: Chanomics International, USAID. $27 \mathrm{p}$.

Johns AD. 1988. Effects of «selective» timber extraction on rain forest structure and composition and some consequences for frugivores and folivores. Biotropica. 20 (1): 31-7.

Jhons AD. 1992. Species conservation in managed tropical forests. En: Whitmore TC, Sayer JA. (Eds.). Tropical deforestation and species extinction. London: Chapman \& Hall. p. 15-53.

Johnson N, Cabarle B. 1993. Surviving the cut: natural forest management in the humid tropics. Washington, DC: World Resources Institute. $71 \mathrm{p}$.

Killeen TJ, Jardim A, Mamani F, Sarava P, Rojas N. 1998. Diversity, composition and structure of a tropical semideciduous forest in the Chiquitanía region of Santa Cruz, Bolivia. J Trop Ecol. 14 (6): 803-27.

Laurance WF, Vasconcelos HL, Lovejoy T. 2001. Forest loss and fragmentation in the Amazon, implications for wildlife conservation. Oryx. 34 (1): 39-45.

Mahecha GE. 1997. Fundamentos y metodología para la identificación de plantas. Proyecto BIOPACÍFICO, Ministerio del Medio Ambiente. PNUD-GEF. 282 p.

Mason DJ. 1996. Responses of venezuelan understory birds to selective logging, enrichment strips, and vine cutting. Biotropica. 28 (3): 296-309.

Mosquera N, Mosquera KD. 2002. Comparación de la estructura y composición florística de dos bosques secundarios pluviales tropicales con diferentes grados de intervención antrópica. (Trabajo de grado). Programa de Biología con énfasis en Recursos Naturales, Universidad Tecnológica del Chocó. 124 p.

Nussbaum R, Anderson RJ, Spencer T. 1995. Factors limiting the growth of indigenous tree seedlings planted on degraded rainforest soils in Sabah, Malaysia. Forest Ecol Manag. 74: 149-59.

Ochoa J. 1997. Sensibilidades potenciales de una comunidad de mamíferos en un bosque productor de maderas de la Guayana venezolana. Interciencia. 22 (3): 112-22.

Ochoa J. 1998. Análisis preliminar de los efectos del aprovechamiento de maderas sobre la composición y estructura de bosques en Guayana venezolana. Interciencia. 23 (4): 197 205.

Ochoa J. 2000. Efectos de la extracción de maderas sobre la diversidad de mamíferos pequeños en bosques de tierras bajas de la Guayana venezolana. Biotropica. 32 (1): 14664.

Ochoa J, Soriano PJ. 2001. The effects of logging on nonvolant small mammal communities in neotropical rain forests. En: Fimbel RA, Grajal A, Robinson JG. (Eds.). The cutting edge: conserving wildlife in logged tropical forest. New York: Columbia University Press. p. 125-52 .

Padmawathe R, Qureshi Q, Rawat GS. 2004. Effects of selective logging on vascular epiphyte diversity in a moist lowland forest of Eastern Himalaya, India. Biol Cons. 119 (1): 8192.

Pinard MA, Howlett B, Davidson D. 1996. Site conditions limit pioneer tree recruitment after logging of dipterocarp forests in Sabah, Malaysia. Biotropica. 28 (1): 2-12.

Plumptre J, Grieser A. 2001. Changes in primate communities following logging disturbance. En: Fimbel RA, Grajal A, Robinson GR. (Eds.). The cutting edge: conserving wildlife in logged tropical forest. New York: Columbia University Press. p. 71-92.

Sarmiento G, Pinillos M, Garay I. 2005. Biomass variability in tropical american lowland reinforests. Ecotropicos. 18 (1): $1-20$.

Soriano PJ, Ochoa J. 2001. The consequences of timber exploitation for bat communities in tropical America. En: Fimbel RA, Grajal A, Robinson JG. (Eds.). The cutting edge: conserving wildlife in logged tropical forest. New York: Columbia University Press. p.153-66.

Thiollay J. M. 1992. Influence of selective logging on bird species diversity in a Guianan rain forest. Biol Cons. 6 (1): 47-63.

Uhl C, Vieira IC. 1989. Ecological impacts of selective logging in the Brazilian Amazon. A case study from the paragominas region of the state of Para. Biotropica. 21 (2): 98-106.

Van Gardingen PR, Clearwater MJ, Nifinluri T, Effendi R, Rusmantoro W, Noor M, et al. 1998. Impacts of logging on the regeneration of lowland dipterocarp forest in Indonesia. Forest Rev. 77 (2): 71-80.

Villareal H, Álvarez M, Córdoba S, Escobar F, Fagua G, Gast F, et al. 2004. Manual de métodos para el desarrollo de inventarios de biodiversidad. Bogotá: Instituto de Investigación de Recursos Biológicos Alexander Von Humboldt.). p. 75-8.

Vitt LJ, Caldwell JP. 2001. The effects of logging on reptiles and amphibians of tropical forests. En: Fimbel RA, Grajal A, Robinson JG. (Eds.). The cutting edge: conserving wildlife in logged tropical forest. New York: Columbia University Press. p. 239-59. 